\title{
Subdermal Atrophy as an Adverse Event after Glucocorticoid- Based Injection Face Modelling
}

\author{
Reznik AV* \\ AR Clinic, Russian Federation
}

*Corresponding author: Anna V Reznik, MD, dermatologist, head physician, Medical Center «ARclinic», Saint-Petersburg, Russian Federation, Email: ksho@yandex.ru

\section{Case Report}

Volume 4 Issue 1

Received Date: March 10, 2020

Published Date: April 20, 2020

DOI: $10.23880 /$ ijtps- 16000141

\section{Abstract}

Subcutaneous injections of glucocorticoids (glucocorticosteroids, GCS) are increasingly used to face modelling. This carries a high risk of soft tissue atrophy.

Aim: This report discusses soft tissue atrophy mechanisms after GCS-injection, describes risk factors and treatment regimens.

Material and methods: The clinical case of treatment of subcutaneous atrophy that occurred after injection of GCS into malar fat pads is presented. Three-stage treatment of atrophy is described.

Results: Atrophy lesion was eliminated as well as volume-deficient facial areas were filled up. The patient was satisfied with the final result.

Conclusions: In aesthetic medicine, GCS should not be applied for intradermal and subcutaneous facial injections due to extremely high risk of dermal and subdermal atrophy.

Keywords: Subdermal atrophy; Lipoatrophy; Glucocorticoids; Corticosteroid injection; Scar; Hypopigmentation; PRP

\section{Introduction}

Currently injection face modelling turned to be more and more popular procedure based on so-called signature or designer mesotherapy cocktails. Patients see mindblowing results of nasal narrowing, malar fat pad correction, chin, cheek surgery, which are all too often produced using advanced photo editing software. Then these patients come to aesthetic medicine professionals doctors with the "do the same to me" request.

It is not uncommon that such signature mesotherapy cocktails for face volume reduction contain glucocorticosteroids (GCS) bearing high risk of soft tissue atrophy after subcutaneous injection. Besides, in most of cases such atrophy develops not immediately post-treatment but in 2 to 16 weeks [1].

Aim: The clinical case of treatment of subcutaneous atrophy that occurred after injection of GCS into malar fat pads is presented. This report discusses soft tissue atrophy mechanisms after GCS, describes risk factors and treatment regimens. My message to aesthetic medicine professionals is that atrophy risk depends just a little bit on physician's injecting skills and in any case remains substantial when such agents are injected in any face area.

\section{Material and methods}

\section{Case Report}

Patient T., female, 35 y.o. The patient came to our clinic one month after corticosteroids (Diprospan) injection in her malar fat pads complaining on tissue atrophy. GCS-injections were performed to reduce malar fat pads. Atrophy emerged 2 weeks post-GCS injection.

\section{At first examination (Figures 1\&2):}

- Dermal and subdermal atrophic lesion with diameter of $2.5 \mathrm{~cm}$ at her right malar area. 


\section{International Journal of Transplantation \& Plastic Surgery}

- Midface soft tissue volume deficiency. Lower eyelid hernias. Marked tear troughs, palpebromalar grooves and nasolabial creases. Notable right malar fat pad.

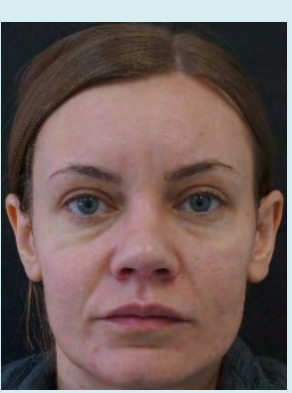

Figure 1: Patient T., female, 35 y.o.: before treatment

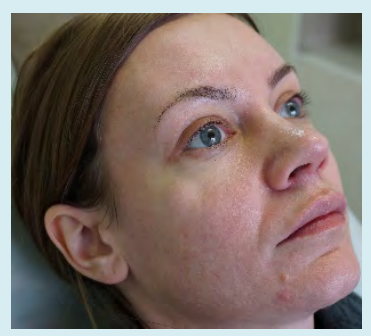

Figure 2: Patient T., female, 35 y.o.: atrophy lesion before treatment.

\section{The patient treatment consisted of 3 stages:}

- $\quad$ Stage 1: Atrophy lesion subcision with 23G needle after preliminary tumescent anesthesia. Single procedure.

- $\quad$ Stage 2:Lesion infiltration with autologous plasma (PRP) aiming to decrease local GCS concentration not only by washing out but also through binding GCS crystals with plasma proteins. Three sessions with 1 week interval were provided. At that stage complete therapeutic effect at the atrophy area was reached.

- Stage 3: Midface injection augmentation. Deficient volume in buccal, malar and periorbital regions and nasolabial folds was restored with hyaluronic acidbased filler.

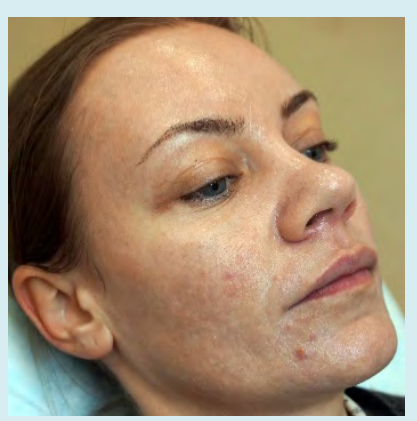

Figure 3: Patient T., female, 35 y.o.: atrophy lesion after PRP-infiltration stage.

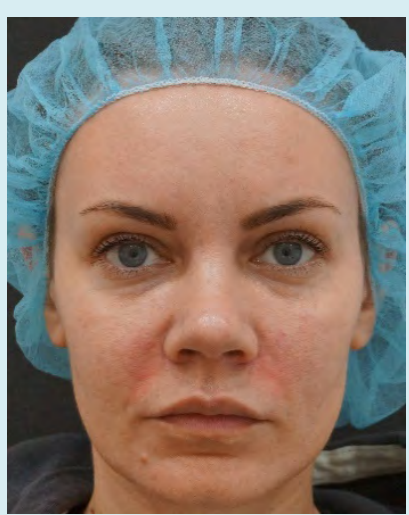

Figure 4: Patient T., female, 35 y.o.: after treatment completion.

\section{Results}

Once the three-stage treatment was finished the atrophy lesion was fully eliminated as well as volume-deficient facial areas were filled up. The patient face turned to look younger and more balanced. She was fully satisfied with the final result.

\section{Discussion}

Soft tissue atrophy mechanisms after subcutaneous injections with Glucocorticosteroids.

GCS subcutaneous injections bear high risk of not only subdermal atrophy but also skin hypopigmentation. Moreover, it develops not only at injection site but along lymphatic vessels as well. Key points for the event:

1. GCS lymphogenic spread:

Glucocorticosteroid molecules are, actually, large crystals. Thus, they cannot advance in blood stream but are able to enter lymphatic system. In lymphatic fluid GCSs bind to albumins and globulins. After GCS injection its concentration at the injection site exceeds lymph binding capacity. Thus, unbound GCS molecules are transferred along lymphatics producing tissue damage, atrophy and hypopigmentation $[1,2]$.

2. Non-immune atrophy mechanism:

Histology studies of atrophy sites show clusters of activated macrophages absorbing damaged fat and stroma fragments. Macrophages are activated directly via tissue trauma by GCS molecules and release a great deal of various cytokines that indirectly leads to atrophy and fibrosis [3].

3. Locally injected GCSs produce vasoconstricting effects bringing forth tissue ischemia and even vascular thrombosis. It prominently increases tissue atrophy risks $[3,4]$.

4. GCS hinders both type I and type III collagen synthesis 


\section{International Journal of Transplantation \& Plastic Surgery}

and degradation, therefore, impairing normal wound healing followed with atrophic scar tissue development [4].

5. GCS suppresses melanocyte function without considerable alteration in their counts that is important for hypopigmentation to develop [4].

Severity and duration of dermal and subdermal atrophy depends on GCS agent type, solubility and concentration (it is higher after triamcinolone and betamethasone injections vs. hydrocortisone ones). Localized subdermal atrophy is seen more frequently in women vs. men (Figures $5 a, 5 b$ ). It develops from 2 weeks to 4 months post-procedure and in many cases resolves without any treatment within 30 months [4]

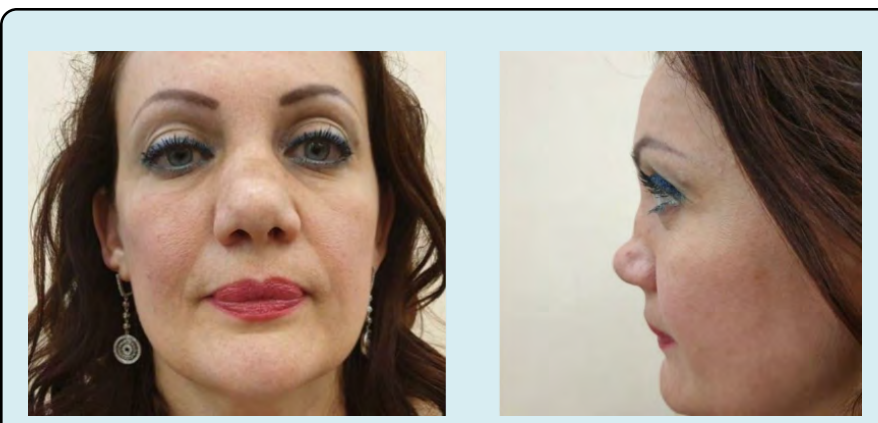

Figure 5a, b: Soft tissue atrophy after Diprospan-based injection rhinoplasty (clinical case image provided by Kazbek Kudzayev, MD, surgeon).

\section{Subdermal atrophy treatment regimen}

A treatment regimen for post-GCS subdermal atrophy consists of 3 stages [5-7].

- Stage 1: Atrophy lesion infiltrated with normal saline Subcutaneous injection of 4 to $20 \mathrm{ml}$ of $0.9 \% \mathrm{NaCl}$ solution in an atrophy lesion once a week aiming to wash out GCS crystals from tissues and to decrease local GCS concentration. Treatment duration - 3 to 6 weeks.

- $\quad$ Stage 2: Treatment with dermal filler injections.

- Stage 3: Structural fat-grafting (lipofilling) is recommended in atrophy cases lasting longer than 1 year.

\section{Conclusions}

In aesthetic medicine, GCS should not be applied for intradermal and subcutaneous facial injections due to extremely high risk of dermal and subdermal atrophy. Despite post-GCS atrophy in many cases disappears without any treatment within 2.5 years this is still the time period such patients should live with facial atrophic scars making their life extremely uncomfortable. Therefore, atrophy treatment is better to be started as early as possible after it appeared combining various aesthetic medicine procedures to generate best possible clinical outcomes.

\section{References}

1. Pace CS, Blanchet NP, Isaacs JE (2018) Soft Tissue Atrophy Related to Corticosteroid Injection: Review of the Literature and Implications for Hand Surgeons. J Hand Surg Am 43(6): 558-563.

2. Prasad K, Madke B, Kar S, Sinha AK, Yadav N (2015) Linear rays of depigmentation along lymphatics after intralesional corticosteroid therapy. Indian dermatology online journal Indian Dermatol Online J 6(6): 456-457.

3. Ahmed I (2006) Post-injection involutional lipoatrophy: ultrastructural evidence for an activated macrophage phenotype and macrophage related involution of adipocytes. Am J Dermatopathol 28(4): 334-337.

4. Oishi Y, Fu ZW, Ohnuki Y, Kato H, Noguchi T (2002) Molecular basis of the alteration in skin collagen metabolism in response to in vivo dexamethasone treatment: effects on the synthesis of collagen type I and III, collagenase, and tissue inhibitors of metalloproteinases. Br J Dermatol 147(5): 859-868.

5. Blugerman G, Schavelzon D, Becker M, Cáceres R, Schavelzon V, et al. (2017) Lipoatrofia por inyección de esteroides-Diagnóstico y tratamiento. Revista Argentina De Cirugía Plástica 23(3): 126-131.

6. Margulies SL, Morris A (2005) Successful treatment of lipoatrophy with normal saline. JAAD Case Rep 1(6): 415-417.

7. Shumaker PR, Rao J, Goldman MP (2005) Treatment of local, persistent cutaneous atrophy following corticosteroid injection with normal saline infiltration. Dermatol Surg 31(10): 1340-1343.

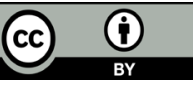

